\title{
Influence of leg length discrepancy on balance and gait in post-stroke patients: a correlational study
}

\author{
Shailesh Gardas * and Hemakshi Shah
}

\begin{abstract}
Background: A frequent complaint by stroke patients presenting for physiotherapy was perceived shortness of the affected lower limb and inability to weight bear onto the affected side. So, the present study aimed to evaluate influence of limb length discrepancy (LLD) on balance and gait parameters in patients with stroke.

Subjects and methods: Twenty participants were recruited based on the inclusion and exclusion criteria and were divided into two groups group $A(L L D \leq 1 \mathrm{~cm})$ and group $B(L L D>1 \mathrm{~cm})$. Postural sway was measured under wide base eyes open, wide base eyes closed, narrow base eyes open, and narrow base eyes closed conditions. Weightbearing asymmetry and functional balance were assessed using the percentage body weight asymmetry and Berg balance scale. Also, step leg ratio and 10-M walk test was used to assess gait parameters.

Results: Intergroup comparison of postural sway exhibited a significant difference between groups in the AP direction in wide-based eyes open (WBEO), wide-based eyes closed (WBEC), narrow-based eyes open (NBEO), and narrow-based eyes closed (NBEC) conditions whereas only in NBEC condition in mediolateral direction; with group $B(L L D>1 \mathrm{~cm})$ displaying a greater mean postural sway in all the conditions. The correlation of LLD with postural sway showed a significant positive correlation within AP direction under all the conditions and in WBEC and NBEC conditions in mediolateral direction. Intergroup comparison of step length ratio (SLR) showed a statistical difference between groups, and a negative correlation was found between LLD and SLR. A negative correlation was also seen between percentage weight-bearing asymmetry (PWBA) and speed as well as Berg's balance scale (BBS), and a positive correlation was observed between BBS and speed.

Conclusion: Leg length discrepancy results in a significant decrease in balance control in the sagittal and frontal planes in patients with stroke. It also adds to the asymmetries in their gait. Therefore, LLD should be considered as a factor for balance and gait asymmetries in post-stroke patients.
\end{abstract}

Keywords: Leg length discrepancy, Postural sway, Weight-bearing asymmetry, Step length ratio, Gait speed

\section{Background}

Stroke or cerebrovascular accident (CVA) as stated by the World Health Organization is a clinical syndrome comprising of rapidly developing clinical signs of focal (or global in case of coma) disturbance of cerebral function lasting more than $24 \mathrm{~h}$ or leading to death with no apparent cause other

\footnotetext{
*Correspondence: shaileshgardas@gmail.com; mgmcopt@hotmail.com; mgm_physiotherapy@yahoo.co.in

Mahatma Gandhi Mission's College of Physiotherapy, Vashi, Navi Mumbai 400705, India
}

\section{Springer Open}

than a vascular origin [1]. It is caused by hypoxia, ischemia, infarction, or intracranial haemorrhage and is the commonest cause of mortality and chronic adult disabilities $[2,3]$. The collective incidence of stroke in India ranged from 105 to $152 / 100,000$ persons per year, and the crude occurrence of stroke ranged from 44.29 to $559 / 100,000$ persons in different parts of the country throughout the past decade [4]. Although $55-70 \%$ of stroke survivors become fully independent by 1 year, $7-15.7 \%$ remained completely disabled [3]. 
In stroke patients, a loss of motor control, abnormal movement patterns, tone disorder [5], muscle weakness [6], sensory dysfunction, and coordination difficulties [7] are some commonly seen deficits especially in the affected lower limbs [6]. The patients also experience difficulties in transferring weight onto the affected side. Asymmetrical weight distribution has been linked to impaired motor function, poor postural control, and standing balance and gait disorders [7-9].

Leg length discrepancy is a bilateral asymmetry of the lower limbs. Functional or apparent LLD can be explained by one-sided asymmetry in the lower extremities, without any associated shortening of the osseous structures $[7,10]$. Several studies done on healthy individual's have shown that simulated discrepancies as little as $5-10 \mathrm{~mm}$ have an effect on the lower limb kinematics by significantly increasing postural sway in normal stance [11]. LLD of sufficient magnitude caused various problems, including increased energy expenditure in gait, equinus contracture of the ankle on the short leg, balance issues, and degenerative joint diseases of the lumbar and hip joints [11, 12]. Additionally, Elkiset et al. in their study found that LLD caused increased asymmetrical weight distribution and an increase in the mean COP velocity suggesting a proportional deterioration of postural sway [13].

A study by Jeon et al. reported that after stroke, patients with hemiplegia exhibit a limb length discrepancy, asymmetry in weight distribution resulting in more imbalance and decreased limits of stability [7]. They argued that the difference in leg length in stroke patients could not only be because of soft tissue contractures of the ipsilateral or contralateral extremity but also angular and torsional deformities. According to them, the contributing factors of LLD could be flexion contractures around the hip and knee joint causing apparent shortening of the leg, whereas equinus deformity and abduction contractures of the hip could lengthen the paretic limb.

Post-stroke patients who present late for physiotherapy usually complaint of perceived shortness of the affected lower limb [14]. Despite this, there is a severe dearth in the literature related to studies on how leg length discrepancy influences various functional outcomes in post-stroke patients. Hence, we intended to explore how leg length discrepancy is associated with various balance and gait parameters in post-stroke patients.

\section{Methods}

This study was an analytical cross-sectional study which commenced with the permission of the institutional ethical committee. Twenty participants were recruited from a tertiary health care hospital in Navi Mumbai by purposive sampling. Patients with chronic stroke within the age group of 30 to 70 years, Brunnstroms stage of recovery III-V, who were able to ambulate at least $10 \mathrm{M}$ independently, a score of $>24$ on Mini Mental Status Examination and patients with LLD were included in this study. Patients presenting with a history of lower limb fractures, total hip replacement, dislocations, visual field defects which could not be corrected, bilateral hemiparesis, cerebellar and brain stem lesions, and unstable neurological conditions like seizures were excluded. Participants were given a detailed explanation of the purpose, procedure of the study in the language best understood by them, and written consent was taken from each.

The apparent leg length was measured using a tape with the patient in a supine position, and measurement was taken from the xiphisternum to the medial malleolus. Two measurements were taken for both the affected and unaffected sides. The average of the values was taken based on which the subjects were then divided into two groups: group $\mathrm{A}-\mathrm{LLD} \leq 1 \mathrm{~cm}$ and group $\mathrm{B}>1$ cm [15, 16] (Fig. 1).

Postural sway was measured using sway meter which was constructed and implemented as per the guidelines given by Sturnieks et al. They conducted a study to assess validity and reliability of the sway meter device and concluded that sway meter is a reliable tool for assessing postural sway [17]. It measures displacements of the body in the horizontal plane at the waist level. The device consisted of an inflexible 40-cm-long rod with a vertically mounted pen at its end. The rod was mounted on a $20-\mathrm{cm}$-wide metal plate which was fitted over the participant's lower back (level of the posterior superior iliac spine) by a firm belt so that the rod extended posteriorly. Fitted firmly, the sway meter offers 1 degree of freedom between the belt and pen as it is free to move in the pitch plane. Sway was measured on a sheet of a millimeter graph paper which was placed behind the participant and secured to the table height using an adhesive tape (Fig. 2). The duration of each trial was $30 \mathrm{~s}$. Three readings were taken one for each condition, i.e., wide base eyes open (WBEO) and eyes closed (WBEC), narrow base eyes open (NBEO), and eyes closed (NBEC). Participants rested for at least $1 \mathrm{~min}$ in between trials. The sway path length was manually determined as the number of millimeter squares traversed by the pen. The antero-posterior (AP) and medio-lateral (ML) peak-topeak sway displacements were also calculated from the extremes of sway length in these two planes. Test-retest reliability of the sway meter was assessed on 30 healthy participants prior to using it in our study. Results showed an excellent reliability in the respective directions in eyes closed condition: ICC of 0.930 (anterior), ICC of 0.899 (posterior), ICC of 0.819 (left lateral), and ICC of 0.917 (right lateral). Whereas in eyes open 


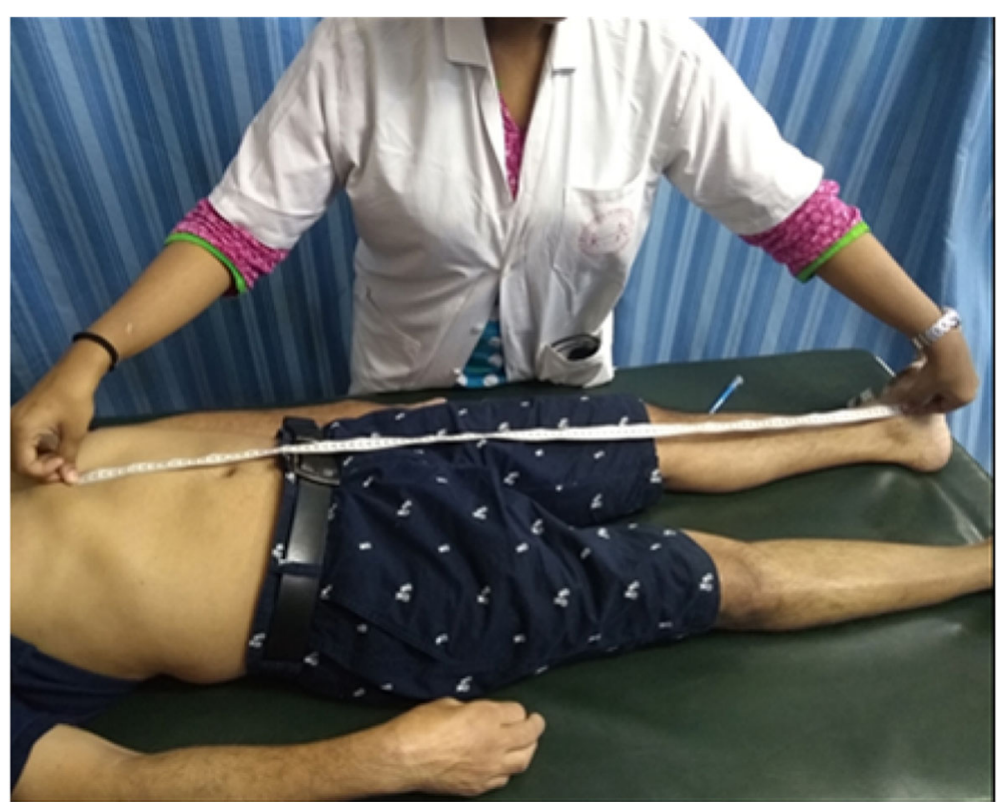

Fig. 1 Measurement of apparent LLD

condition, good to excellent reliability was seen in following directions: ICC of 0.748 (posterior), ICC of 0.887 (left lateral), and ICC of 0.913 (right lateral) except in the anterior ICC of 0.462 .

A digital weighing scale and a wooden block of the same dimension were used for the assessment of the percentage weight-bearing asymmetry (PWBA). First, the total body was calculated using the weighing scale (Fig. 3). To measure the weight bearing during the assessment, the participant stood in such a way that his/ her affected leg was on the platform of the scale while the unaffected leg was on a wide wooden block adjacent to the platform. Then, the measurements were repeated for the unaffected side. Three measurements were repeated and documented. PWBA was calculated using the formula:

PWBA $=[$ (weight on the unaffected leg - weight on the affected leg)/total body weight] $\times 100$ [18].

The footprint method was used to assess the step length. The participants were made to sit on a chair after which their feet were damped and later dipped in a colored chalk powder and were instructed to walk on the walkway made from a long white sheet of paper at a self-selected pace (Fig. 4). An average of 5 steps was taken for both sides. The step length $(\mathrm{cm})$ was measured from the geometrical heel center of the current footprint

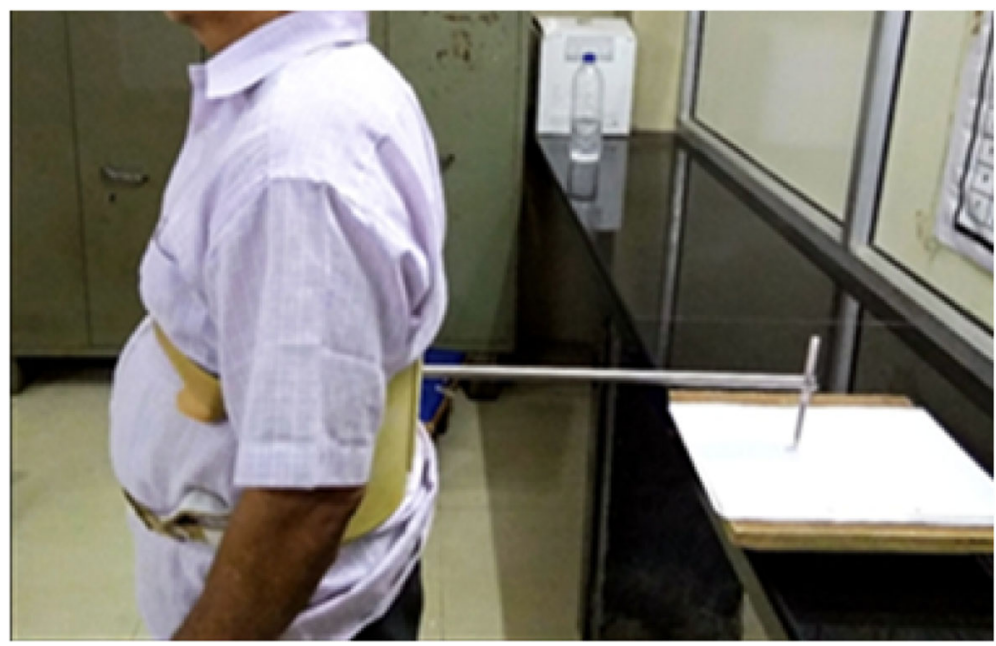

Fig. 2 Measuring postural sway using a sway meter 


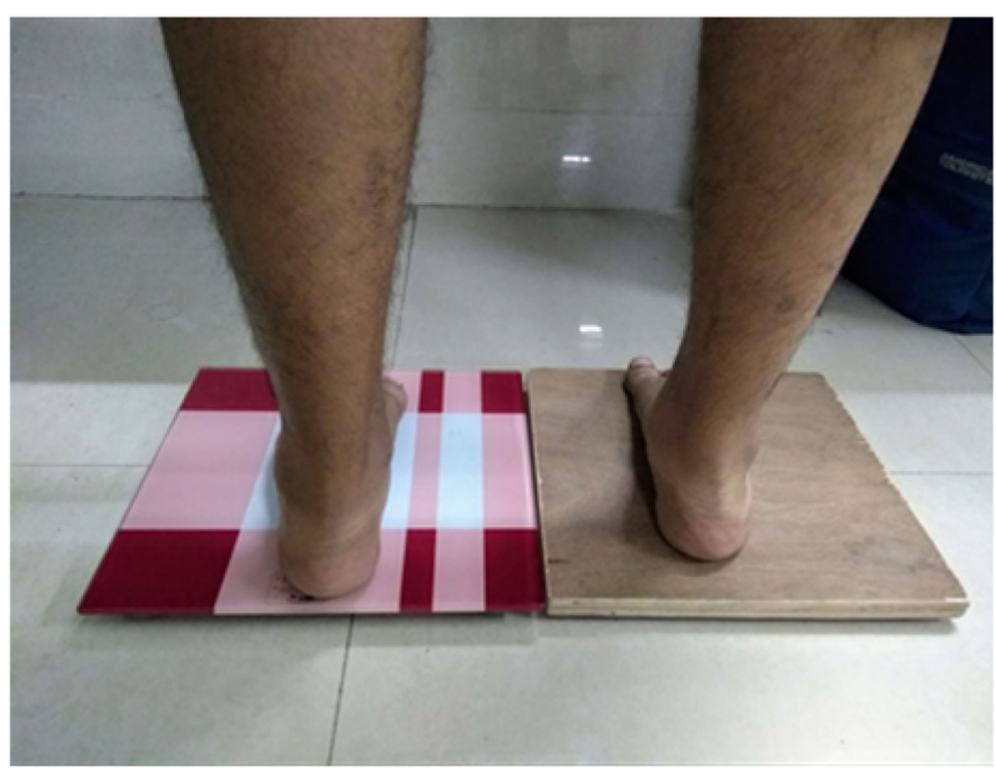

Fig. 3 Measuring weight-bearing asymmetry using digital weighing scale

to the same of the previous footprint on the opposite foot $[19,20]$. The step length symmetry was assessed using step length ratio (SLR) which was defined as a paretic step length divided by non-paretic step length. Step length asymmetry was characterized by 3 patterns. The symmetrical group was defined as participants with an SLR between 0.9 and 1.1. Asymmetrical groups were those with SLR greater than 1.1 (longer paretic steps than non-paretic) and SLR less than 0.9 (longer nonparetic steps than paretic) [21].

Gait speed was measured by a $10-\mathrm{M}$ walk test, where the patients walked at a self-selected pace. The individual was made to walk without assistance for $10 \mathrm{M}$, with the time measured for intermediate $6 \mathrm{M}$ to allow for acceleration and deceleration. A stopwatch was started when the toes pass the 2-M mark and stopped when the toes pass 8-M mark. Participants were given the following instructions: "I will say ready set go. When I say go, walk at your normal comfortable speed until I say stop". Subjects' self-selected walking speed was categorized as speed less than $0.4 \mathrm{~m} / \mathrm{s}$ (household ambulatory), 0.4 to $0.8 \mathrm{~m} / \mathrm{s}$ (limited community), or speed greater than 0.8 $\mathrm{m} / \mathrm{s}$ (community ambulatory) [21].

The balance was assessed with the Berg balance scale (BBS) which is used to objectively determine a patient's ability (or inability) to safely balance during a series of predetermined tasks. It is a 14-item list with each item consisting of a five-point ordinal scale ranging from 0 to 4 , with 0 indicating the lowest level of function and 4 the highest level of function, and has been shown to be a valid and reliable assessment of balance for individuals with chronic stroke [22].

\section{Statistical analysis}

Collected data were entered and analyzed using SPSS statistical software version 17 (SPSS Inc. Chicago, IL, USA). The normality of the data was assessed using the Shapiro-Wilk's test. The correlation of LLD with balance and gait parameters was assessed using the Pearson's correlation coefficient as the data was normally distributed and an independent $t$ test was used to assess intergroup comparison of balance and gait parameters. The level of significance was set at $<0.05$ for all the tests.

\section{Results}

The mean \pm SD of patients' characteristics and duration of illness of groups A and B are demonstrated in Table 1.

Table 2 shows intergroup comparison of postural sway, PWBA, BBS, SLR, and speed was done using the independent $t$ test and was found to be statistically significant for postural sway in the AP direction under the conditions WBEO, WBEC, NBEO, NBEC, and in the $M L$ direction under NBEC with group $B$ having greater mean values. There was also a statistically significant difference in the SLR, $p$ value 0.007 with group A having a greater mean value. Comparisons were statistically insignificant for PWBA, BBS, and speed.

In this study, (Table 3) LLD with postural sway showed a moderate to strong positive correlation within AP direction under the conditions WBEO, WBEC, NBEO, and NBEC, and a moderate positive to strong positive correlation was also seen with postural sway in the medio-lateral direction but was observed under the conditions WBEC and NBEC. A moderate negative correlation was found between LLD and SLR $(p<0.05)$ 


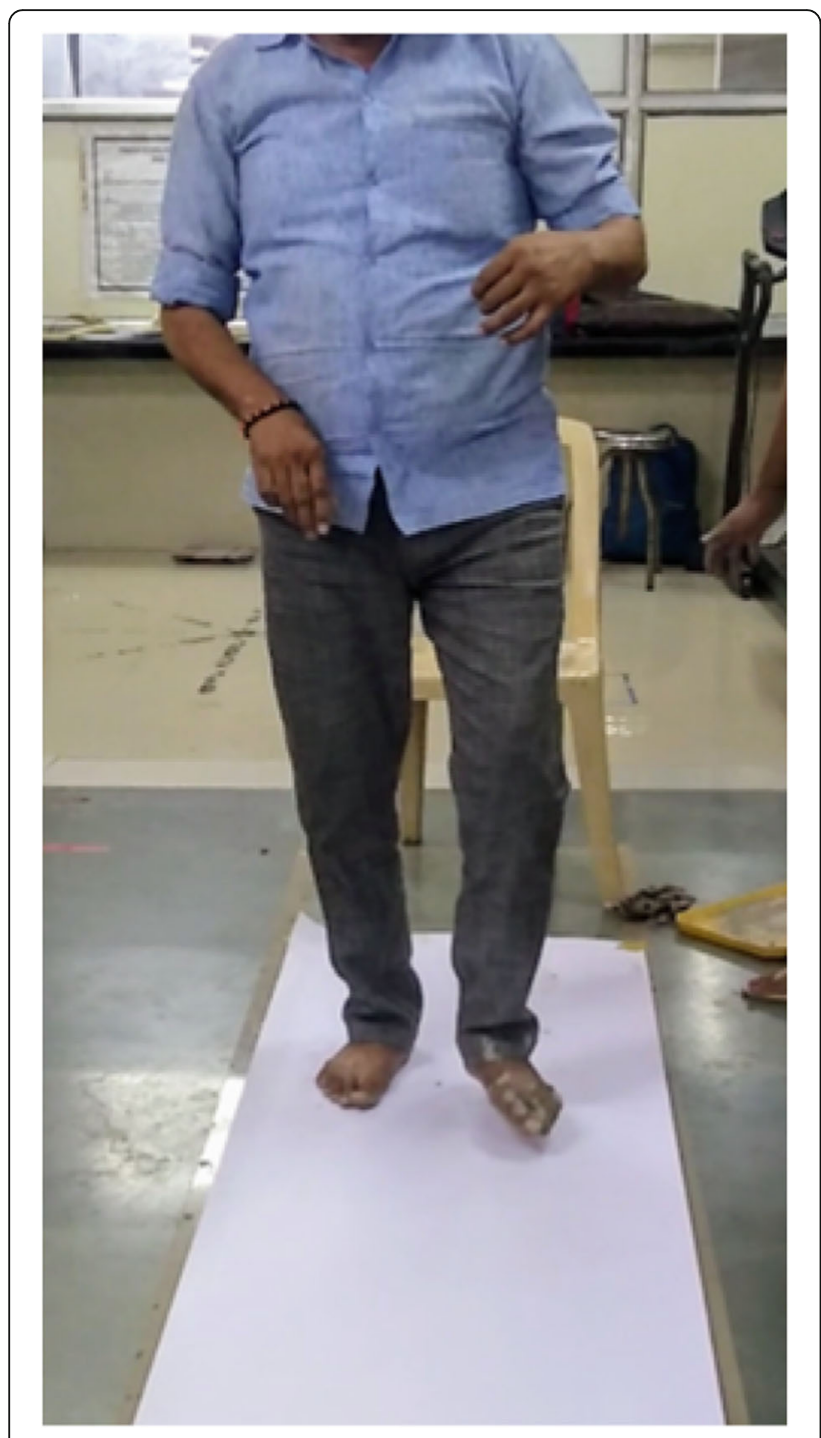

Fig. 4 Measuring step length using the foot print method

Table 1 Baseline characteristics of all participants allocated in group A and group B

\begin{tabular}{lll}
\hline & Group A [N (\%)] & Group B [N (\%)] \\
\hline $\begin{array}{l}\text { Age (years) } \\
\text { Duration of illness (months) }\end{array}$ & $39.9 \pm 12.56^{*}$ & $52.3 \pm 10.78^{*}$ \\
Sex & & $26.20 \pm 28.00^{*}$ \\
$\quad$ Male/female & $4(40 \%) / 6(60 \%)$ & $10(100 \%) /-$ \\
$\begin{array}{l}\text { Types of stroke } \\
\quad \text { Ischemic }\end{array}$ & $5(70 \%)$ & $5(50 \%)$ \\
Hemorrhagic & $3(30 \%)$ & $5(50 \%)$ \\
Affected side & & $2(20 \%)$ \\
Right & $5(50 \%)$ & $8(80 \%)$ \\
$\quad$ Left & $5(50 \%)$ & \\
\hline
\end{tabular}

*Expressed as mean \pm S.D
There was no significant correlation found between LLD, PWBA, BBS, and speed. However, there was a moderate positive correlation between PWBA and BBS, PWBA and speed, SLR and speed, and a strong positive correlation between BBS and speed $(p<0.05)$.

\section{Discussion}

This study aimed to explore the association of the leg length discrepancy with balance parameters like postural sway, percentage of weight-bearing asymmetry, Berg balance score, and gait parameters like the step length asymmetry ratio and speed in patients following stroke.

In this study, intergroup comparison revealed a significant difference in the AP sway under all the balance conditions and in ML sway under the condition NBEC. It was seen that group B (LLD $>1 \mathrm{~cm})$ had a greater mean postural sway in all the conditions suggesting that higher LLD was more challenging for the subjects. Also, the correlation of LLD with postural sway showed a moderate to strong positive correlation in AP direction under all the conditions and in ML direction under the conditions WBEC and NBEC which means, as the LLD increased the sway also increased exponentially.

Altered postural sway in patients with stroke could be dependent on numerous factors such as reduced joint proprioception, sensory deficits, reduced motor coordination, reduced joint range of motion, weakness, spasticity, and/or attention deficit [23]. Studies have shown that the incidence of spasticity among paretic patients has been reported to be $27 \%$ at 1 month, $28 \%$ at 3 months, $23 \%$ and $43 \%$ at 6 months, and $34 \%$ at 18 months after stroke $[24,25]$. In addition, they suggested that neuronal components of spasticity peak at 3 months after stroke, the muscular components of spasticity may increase over time contributing to increased incidence of spasticity at 6 months post-stroke. In the current study, since the participants were in the chronic recovery stage of the disease, they displayed a mix of spasticity and tightness.

Muscle spasticity post stroke is resultant of disinhibition and hyperextitability of the descending pathways primarily VST \& RST, whereas the muscle weakness occurs due to the damage to the corticospinal tracts. Spasticity coupled with weakness causes impaired voluntary control and muscle activation in the lower limb muscles causing abnormal synergistic activity [26]. In chronic stroke patients, the prolonged spasticity and weakness leads to contractures of the soft tissues which coupled with abnormal synergies could result in LLD. In the present study, all the participants had the presence of spasticity on assessment using Modified Ashworth's Scale (MAS). It was seen in the following lower limb muscles: hip adductors, knee flexors, knee extensors, and gastrosoleus. The grade of spasticity ranged from $1+$ 
Table 2 Intergroup Comparison of Balance and gait parameters

\begin{tabular}{|c|c|c|c|c|}
\hline \multicolumn{2}{|c|}{ Outcome measures } & $\begin{array}{l}\text { Mean } \pm \text { SD }(\mathrm{mm}) \\
\text { Group A }\end{array}$ & $\begin{array}{l}\text { Mean } \pm \text { SD }(\mathrm{mm}) \\
\text { Group B }\end{array}$ & \multirow{2}{*}{$\begin{array}{l}p \text { value } \\
\text { Independent } t \text { test }\end{array}$} \\
\hline \multirow{9}{*}{ Postural sway } & & & & \\
\hline & WBEO AP & $10.35 \pm 2.33$ & $18.11 \pm 7.83$ & $0.007^{*}$ \\
\hline & WBEC AP & $12.20 \pm 3.80$ & $23.50 \pm 7.90$ & $0.001^{*}$ \\
\hline & NBEO AP & $14.39 \pm 5.06$ & $22.14 \pm 5.48$ & $0.004^{*}$ \\
\hline & NBEC AP & $18.35 \pm 6.88$ & $31.04 \pm 13.07$ & $0.040^{*}$ \\
\hline & NBEC ML & $26.54 \pm 7.89$ & $27.79 \pm 11.61$ & $0.007^{*}$ \\
\hline & WBEO ML & $14.09 \pm 4.96$ & $19.76 \pm 12.18$ & 0.190 \\
\hline & WBEC ML & $15.46 \pm 5.02$ & $22.44 \pm 5.48$ & 0.171 \\
\hline & NBEO ML & $23.09 \pm 31.04$ & $6.88 \pm 11.61$ & 0.082 \\
\hline \multicolumn{2}{|l|}{ PWBA } & $13.57 \pm 7.47$ & $20.14 \pm 11.16$ & 0.139 \\
\hline \multicolumn{2}{|l|}{ Step leg ratio } & $0.83 \pm 0.22$ & $0.63 \pm 0.12$ & $0.007^{*}$ \\
\hline \multicolumn{2}{|l|}{ BBS } & $46.50 \pm 4.97$ & $47.40 \pm 2.91$ & 0.627 \\
\hline \multicolumn{2}{|l|}{ Speed } & $0.49 \pm 0.134$ & $0.51 \pm 0.18$ & 0.778 \\
\hline
\end{tabular}

$A P$ antero-posterior, $M L$ medio-lateral, WBEO wide base eyes open, WBEC wide-based eyes closed, NBEC narrow-based eyes open, NBEC narrow-based eyes closed *Indicates $p$ value $<0.05$

to 3, gastrosoleus being the most spastic amongst all the muscles. Most of the participants also demonstrated tightness of knee flexors, gastrosoleus, and pelvic mal alignment due to overuse of hip hikers. These could be few of the contributing factors of LLD resulting in gait and balance impairments.

Dickstein et al. in their study found an increase in postural sway in AP direction during stance in patients with stroke. He stated that sway at the waist level is higher compared to the leg level with its axis at ankle joints.

Table 3 Depicts association between LLD, balance, and gait outcome measures

\begin{tabular}{lll}
\hline Outcomes & Pearson's correlation coefficient & $\boldsymbol{p}$ values \\
\hline WBEO AP with LLD & 0.489 & $0.029^{*}$ \\
WBEC AP with LLD & 0.681 & $0.001^{*}$ \\
NBEO AP with LLD & 0.450 & $0.047^{*}$ \\
NBEC AP with LLD & 0.658 & $0.002^{*}$ \\
WBEC ML with LLD & 0.449 & $0.047^{*}$ \\
NBEC ML with LLD & 0.646 & $0.002^{*}$ \\
SLR with LLD & -0.540 & $0.014^{*}$ \\
PWBA with LLD & 0.238 & 0.312 \\
BBS with LLD & 0.014 & 0.954 \\
Speed with LLD. & 0.067 & 0.778 \\
PWBA with speed & -0.446 & $0.042^{*}$ \\
PWBA with BBS & -0.459 & $0.042^{*}$ \\
BBS with speed & 0.810 & $0.000^{*}$ \\
PSL with speed. & 0.502 & $0.024^{*}$ \\
\hline
\end{tabular}

SLR step length ratio, PWBA percentage weight-bearing asymmetry, BBS Berg's balance scale, LLD leg length discrepancy, PSL paretic step length

*Indicates $p$ value $<0.05$
This finding is in accordance to the inverted pendulum model which could be due to the combined use of ankle and hip strategies to control AP sway [27]. Additionally, it is seen that stroke patients exhibited an inability to shift weight to the paretic side and weight bear more on non-paretic side due to several reasons like visuospatial deficit, contracture, spasticity, impaired sensation, weakness, and fear of fall [2]. This could cause a reduced medio-lateral sway in them. Patients with stroke also show a reduced attention capacity and a tendency to fall towards the paretic side [2, 28]. This fear of the fall and risk of injuries could be another reason that stroke patients put in conscious efforts to maintain their balance reducing sway in the ML direction until they are put into more challenging conditions like NBEC, which poses a greater challenge to stability and imbalance, as seen in the present study.

Intergroup comparison of PWBA, BBS, and speed did not show a significant difference amongst them nor did it reveal any significant correlation with LLD. Our findings are contradictory to the results of a study by Jeon et al. They reported that patients with hemiparesis could become more unbalanced because of asymmetry of weight distribution [7]. In the current study, even though we observed a decreased weight bearing on the affected side compared to the unaffected side, it was found to be statistically insignificant (PWBA mean and S.D of group A $13.57 \pm 7.47$ and group B $20.14 \pm 11.16$ ). The participants that were included were functionally ambulatory, independent, and were in the chronic phase of the disease (average duration of condition group A-32.4 months and group B-26.2 months). The chronicity of the disease might have resulted in self adaptation and development of compensatory strategies to overcome the asymmetry. 
Intergroup comparison for SLR showed a significant difference between groups as seen by decreased SLR with an increase in LLD in group B as compared to group $\mathrm{A}$. In addition to this, a negative correlation was found between LLD and SLR that means, as the LLD increased, there was a decrease in the SLR. The group with LLD more than $1 \mathrm{~cm}$ exhibits a shorter paretic step length. This could be due to weakness of musculature, abnormal voluntary control of the paretic limb, poor motor coordination, impaired proprioception, and restricted mobility due to stiffness. So, to maintain the speed of walking and progression of gait, the participants exhibited a longer non-paretic step length to compensate [29].

While SLR was found to have no association with speed in our study, a moderate positive correlation was observed between paretic step length (PSL) and speed. Our findings were supported by results of study conducted by Balasubramanian et al. They reported a weak relationship between step length asymmetry and walking speed in patients with chronic hemi paresis, indicating that asymmetrical patterns need not necessarily limit the attained walking speed [21]. In this study, out of the 20 participants, only four demonstrated symmetrical step length, i.e., SLR between 0.9 and 1.1. Hence, we observed that most of the participants had a walking speed that fell between 0.4 and $0.8 \mathrm{~m} / \mathrm{s}$ (which indicates household to limited community ambulatory).

In the present study, PWBA had a moderate negative correlation with BBS, i.e., as the weight-bearing asymmetry increases, the Berg balance score decreases. Weight-bearing asymmetry is linked to poor postural stability, falls, and gait disorders which increases with dual-task performances and is also a cause for poor performance of functional activities while standing [9]. Chengetanai et al. in their study observed that as the asymmetry in weight distribution increases between the limbs, there was a decrease in scores of BBS with movement activities. This was attributed to an inability of the patients to transfer weight (weight shifts) onto either the limb which is very essential for smooth and efficient execution of activities of daily living involving balance and movement [18, 30].

A negative correlation was observed between PBWA and speed. Andrzej et al. tried to find out link between weight shift asymmetry and gait disturbances in chronic hemiparetic post stroke patients. They observed a significant negative association between weight-bearing asymmetry, paretic step length, and walking speed. The probable explanation for these findings could be kinematic deviations in gait such as asymmetrical movement of the pelvis, inadequate paretic hip and knee flexion in swing phase, and excessive ankle plantar flexion that affect the spatiotemporal parameters [30]. Furthermore, Adegoke et al. in a study evaluating asymmetry of weight bearing on the lower limbs and the association between PWBA and functional ambulation performance in ambulant stroke survivors found a strong correlation between the outcomes [18].

BBS had a strong positive correlation with speed which means as the BBS score increased, the gait speed increased. A higher score was indicative of a good functional balance. Individuals presenting with higher score represented an improved ability to carry out movements with good weight transference and better motor function, thus better-walking speed. Similar results were observed in a pilot study by Kobayashi et al. on patients with chronic stroke [31]. Another study by Britto et al. also reported correlations between static and functional balance, gait speed, and walking ability. They also suggested that balance training might be an important component for gait recovery protocols [32].

The leg length discrepancy as seen in the present study challenges the balance and gait in post stroke patients who already exhibit poor balance and gait asymmetries which could further predispose them to fear and risk of falls or injuries. Thus, prevention of occurrence of LLD should be achieved by educating the patients about the ill effects of LLD, regular monitoring of changes in the limb length by therapist, and focusing rehabilitation on the functional impairments such as spasticity, tightnesss, and abnormal motor control, which are considered to be contributory factors of LLD. Studies on how compensatory strategies such as modifying height of patient's footwear or adjusting the height of orthotic devices could address LLD must be undertaken in future. Further studies are needed to create neurophysiotherapies through objective measurements of the asymmetric pelvis and leg length discrepancy in hemiplegic patients after stroke.

There were some limitations in this study. First, the probable reasons for LLD such as severity of tonal abnormalities, chronicity of condition, etiology of stroke, and variability in duration of rehabilitation were not considered individually in the study. Future studies assessing and evaluating these factors could have given us an in-depth understanding of the specific causes of LLD and resultant balance and gait limitations. Secondly, the sample size of the study was small. Studies done with a larger sample would be better for generalizing the findings. Lastly, we used a self-constructed device to assess postural sway due to a lack of resources and infrastructure. A more accurate, robust, and gold standard method such as VICON, a system that offers motion analysis, would have given a more reliable and accurate insight into the outcomes. 


\section{Conclusion}

The leg length discrepancy in patients with stroke results in significant decrease in a balance control in a sagittal plane under all the conditions and in the frontal plane in more challenging conditions like the eyes closed and narrow base. It also adds to the asymmetries in gait parameters. Therefore, LLD should be considered as a key factor affecting balance and gait parameters like step length ratio in stroke and should be routinely examined so that necessary interventions could be given to avoid deleterious consequences.

\section{Abbreviations}

LLD: Leg length discrepancy; AP: Antero-posterior; ML: Medio-lateral; WBEO: Wide base eyes open; WBEC: Wide base eyes closed; NBEO: Narrow base eyes open; NBEC: Narrow base eyes closed; SLR: Step length ratio; PSL: Paretic step length; PWBA: Percentage of weight-bearing asymmetry; BBS: Bergs balance scale score; COM: Centre of mass

\section{Acknowledgements}

The authors acknowledge the patients for their participation and cooperation in the study.

\section{Authors' contributions}

SG has contributed to the concept, design, literature search, data analysis, manuscript preparation, editing, and review. HS has contributed to the design, literature search, constructing the sway meter, data collection, and data analysis and manuscript preparation. The authors have read and approved the final manuscript.

\section{Funding}

The authors received no financial support for the research, authorship, and/ or publication of this article.

\section{Availability of data and materials}

The datasets used and/or analyzed during the current study are available from the corresponding author on reasonable request.

\section{Ethics approval and consent to participate}

The Institutional Research Review Committee, Mahatma Gandhi Mission's, College of Physiotherapy, Navi Mumbai (Reference no: MGM/COP/IRRC/ 1220/2018), approved the study. The subjects participating in the study were given a detailed explanation of the purpose and the procedures of the study in the language best understood by them. Written consent was taken from each participant in the language understood by them before commencing the study.

\section{Consent for publication}

The participants consented to the use of their details (pictures) for this study.

\section{Competing interests}

The authors declare no potential conflicts of interest with respect to the research, authorship, and/or publication of this article.

Received: 23 July 2020 Accepted: 1 October 2020

Published online: 18 November 2020

\section{References}

1. Chugh C. Acute ischemic stroke: management approach. Indian J Crit Care Med. 2019;23(S2):140-6. https://doi.org/10.5005/jp-journals-10071-23192.

2. Yu J, Jung J, Cho K. Changes in postural sway according to surface stability in post-stroke patients. J Phys Ther Sci. 2012;24(11):1183-6.

3. Banerjee T, Das S. Fifty years of stroke researches in India. Ann Indian Acad Neurol. 2016:19(1):1.

4. Kamalakannan S, Gudlavalleti AV, Gudlavalleti VM, Goenka S, Kuper H. Incidence \& prevalence of stroke in India: a systematic review. Indian J Med Res. 2017;146(2):175
5. Moon S-H, Choi J-H, Park S-E. The effects of functional electrical stimulation on muscle tone and stiffness of stroke patients. J Phys Ther Sci. 2017:29(2): 238-41

6. Lee W-D, Kim J-H, Lee J-U, et al. Differences in rheobase and chronaxie between the paretic and non-paretic sides of hemiplegic stroke patients: a pilot study. J Phys Ther Sci. 2013;25(6):717-9.

7. Jeon $\mathrm{H}-\mathrm{J}$, Kim M-Y, Lee J-U, Kim J. Differences in leg length discrepancy and weight distribution between the healthy and unhealthy sides of hemiplegic stroke patients. Toxicol Environ Health Sci. 2013;5(4):221-6.

8. Sackley CM. The relationships between weight-bearing asymmetry after stroke, motor function and activities of daily living. Physiother Theory Pract. 1990;6(4):179-85.

9. Kamphuis JF, de Kam D, Geurts ACH, Weerdesteyn V. Is weight-bearing asymmetry associated with postural instability after stroke? A systematic review. Stroke Res Treat. 2013:2013:1-13.

10. Gurney B. Leg length discrepancy. Gait Posture. 2002;15(2):195-206.

11. Khamis S, Carmeli E. The effect of simulated leg length discrepancy on lower limb biomechanics during gait. Gait Posture. 2018;61:73-80.

12. Murray KJ, Molyneux T, Le Grande MR, Castro Mendez A, Fuss FK, Azari MF. Association of mild leg length discrepancy and degenerative changes in the hip joint and lumbar spine. J Manipulative Physiol Ther. 2017:40(5):320-9.

13. Eliks M, Ostiak-Tomaszewska W, Lisiński P, Koczewski P. Does structural leglength discrepancy affect postural control? Preliminary study. BMC Musculoskelet Disord. 2017;18(1):346

14. Achugbue FS, Sanusi S. Post-stroke lower limb length discrepancy: a complication in patients with hemiparesis. Res J Med Sci. 2009;3(5):175-8.

15. Hoyle DA, Latour M, Bohannon RW. Intraexaminer, interexaminer, and interdevice comparability of leg length measurements obtained with measuring tape and metrecom. J Orthop Sport Phys Ther. 1991;14(6):263-8.

16. Neelly K, Wallmann HW, Backus CJ. Validity of measuring leg length with a tape measure compared to a computed tomography scan. Physiother Theory Pract. 2013;29(6):487-92. https://doi.org/10.3109/09593985.2012. 755589

17. Sturnieks DL, Arnold R, Lord SR. Validity and reliability of the swaymeter device for measuring postural sway. BMC Geriatr. 2011;11(1):63.

18. Adegoke BOA, Olaniyi $\mathrm{O}$, Akosile $\mathrm{CO}$. Weight bearing asymmetry and functional ambulation performance in stroke survivors. Glob J Health Sci. 2012:4(2):87-94.

19. Wilkinson MJ, Menz HB. Measurement of gait parameters from footprints: a reliability study. Foot. 1997:7(1):19-23.

20. Kaur S, Singh V. Quantitative gait analysis of healthy adults using foot print method and win track. IOSR J Nurs Heal Sci. 2014;3:16-21.

21. Balasubramanian CK, Bowden MG, Neptune RR, Kautz SA. Relationship between step length asymmetry and walking performance in subjects with chronic hemiparesis. Arch Phys Med Rehabil. 2007:88(1):43-9.

22. Alzayer $L$, Beninato $M$, Portney $L G$. The accuracy of individual berg balance scale items compared with the total berg score for classifying people with chronic stroke according to fall history. J Neurol Phys Ther. 2009;33(3):136-43.

23. Bensoussan L, Viton J-M, Schieppati M, et al. Changes in postural control in hemiplegic patients after stroke performing a dual task. Arch Phys Med Rehabil. 2007:88(8):1009-15.

24. Wissel J, Manack A, Brainin M. Toward an epidemiology of poststroke spasticity. Neurology. 2013:80(Issue 3, Supplement 2):S13-9.

25. Opheim A, Danielsson A, Alt Murphy M, Persson HC, Sunnerhagen KS. Upper-limb spasticity during the first year after stroke. Am J Phys Med Rehabil. 2014;93(10):884-96.

26. Li S, Francisco GE, Zhou P. Post-stroke hemiplegic gait: new perspective and insights. Front Physiol. 2018;9.

27. Dickstein R, Abulaffio N. Postural sway of the affected and nonaffected pelvis and leg in stance of hemiparetic patients. Arch Phys Med Rehabil. 2000;81(3):364-7.

28. Farahmand B, Ebrahimi Takamjani E, Yazdi HR, Saeedi H, Kamali M, Bagherzadeh CM. A systematic review on the validity and reliability of tape measurement method in leg length discrepancy. Med J Islam Repub Iran. 2019;33:46.

29. Allen JL, Kautz SA, Neptune RR. Step length asymmetry is representative of compensatory mechanisms used in post-stroke hemiparetic walking. Gait Posture. 2011:33(4):538-43.

30. Szopa A, Domagalska-Szopa M, Lasek-Bal A, Żak A. The link between weight shift asymmetry and gait disturbances in chronic hemiparetic stroke patients. Clin Interv Aging. 2017;12:2055-62. 
31. Kobayashi T, Leung AKL, Akazawa Y, Hutchins SW. Correlations between Berg balance scale and gait speed in individuals with stroke wearing ankle-foot orthoses - a pilot study. Disabil Rehabil Assist Technol. 2016;11(3):219-22.

32. Britto HM, Mendes LD, Moreno CD, Lindquist AR. Correlation between balance, speed, and walking ability in individuals with chronic hemiparesis. Fisioter em Mov. 2016;29(1):87-94.

\section{Publisher's Note}

Springer Nature remains neutral with regard to jurisdictional claims in published maps and institutional affiliations.

Submit your manuscript to a SpringerOpen ${ }^{\circ}$ journal and benefit from:

- Convenient online submission

- Rigorous peer review

- Open access: articles freely available online

- High visibility within the field

- Retaining the copyright to your article

Submit your next manuscript at $\boldsymbol{\sim}$ springeropen.com 